\title{
Neuropsychiatric effects of tenofovir in comparison with other antiretroviral drugs
}

This article was published in the following Dove Press journal:

Neurobehavioral HIV Medicine

21 January 2013

Number of times this article has been viewed

\author{
Kathleen Ferrer ${ }^{1,2}$ \\ Natella Rakhmanina ${ }^{1,2}$ \\ 'Department of Pediatrics, George \\ Washington University School of \\ Medicine, Washington, DC, USA; \\ ${ }^{2}$ Health Sciences Children's National \\ Medical Center, Washington, DC, USA
}

\begin{abstract}
Tenofovir is a widely used antiretroviral medication indicated to treat adults and children infected with HIV. Current guidelines for the management of HIV infection recommend tenofovir disoproxil fumarate (TDF) as a component of the preferred first-line combination antiretroviral therapy. The efficacy, tolerability, prolonged half-life allowing for once-daily administration, and availability as a component of several fixed-dose formulations make TDF an attractive choice for treatment-naive and treatment-experienced HIV-infected patients. TDF is also widely used as a component of postexposure prophylaxis in noninfected individuals. Most importantly, it has been recently approved for use as pre-exposure prophylaxis for noninfected adults and adolescents to reduce the risk of HIV transmission. With increasing use of TDF among adults and children, understanding of the potential for drug-associated side effects is important. This review focuses on the neuropsychiatric effects of tenofovir in adults and children with HIV infection in comparison with other antiretroviral drugs.
\end{abstract}

Keywords: HIV, tenofovir, neuropsychiatric effects

\section{Introduction}

Antiretroviral therapy (ART) has transformed the landscape of HIV medicine. Since the introduction of combination ART (cART) in the mid-nineties, the morbidity and mortality of patients with HIV infection has decreased dramatically. ${ }^{1,2}$ Combination of three or more antiretroviral (ARV) medications is required to achieve and maintain control of viral replication. ${ }^{1}$ Currently, over 25 individual ARV medications are approved by the Food and Drug Administration (FDA) to treat HIV-infected patients in the United States. Knowledge about efficacy and toxicity of ARV drugs is crucial in designing the most potent, tolerable, and convenient ART to optimize outcome of HIV therapy.

Since its introduction, tenofovir (TFV) disoproxil fumarate (DF) has become an important component of cART in adolescents and adults. Tenofovir disoproxil fumarate (TDF) was approved by the US FDA for the treatment of HIV-1 infection in 2001 for adults and children older than 12 years of age. In January 2012, TDF was approved by the FDA for use in pediatric patients $>2$ years of age and in July of 2012, the TDF coformulation with emtricitabine (FTC), Truvada (Gilead Sciences, Foster City, CA, USA), became the first drug approved by the FDA for use in noninfected adolescents and adults to reduce the risk of sexually acquired HIV infection as part of pre-exposure prophylaxis (PrEP).

Several clinical trials have demonstrated high efficacy of TDF in suppressing viral replication in both treatment-naive and treatment-experienced adult, adolescent, and 
pediatric patients. ${ }^{3,4}$ TDF has higher antiviral potency compared with other nucleoside reverse-transcriptase inhibitors (NRTIs) with the median decrease in plasma HIV RNA in subjects receiving TDF, zidovudine (AZT), or stavudine (d4T) monotherapy being $1.4,0.5$, and $0.5 \log _{10}$, respectively. ${ }^{5}$ Pediatric studies have equally demonstrated excellent virologic response to TDF. ${ }^{6-8}$ Due to its efficacy, safety profile, and tolerability, TDF has been chosen as a component of preferred ART recommended as initial HIV treatment by the Department of Health and Human Services (DHHS) and the World Health Organization (WHO) guidelines for antiretroviral-naive adults and children (Table 1). ${ }^{9-11}$

Additionally, in 2011 the HPTN 052 trial conducted by the HIV Prevention Trials Network in the US, showed that use of a TDF-containing regimen in an HIV-positive partner resulted in a $96 \%$ reduction in transmission risk. ${ }^{12}$ This study formed the basis for the concept of treatment as prevention, which has subsequently led to several studies investigating the safety and efficacy of using a TDF-containing regimen in noninfected individuals for PrEP.

With increasing use of TDF among adults and children, understanding of the potential for drug-associated side effects is important. This article will review the neuropsychiatric effects of TDF in comparison with other ARV drugs.

\section{Tenofovir in clinical practice}

Classified as an nucleotide reverse-transcriptase inhibitor (NtRTI), TDF is a prodrug that is converted to TFV, an acyclic nucleoside phosphonate (nucleotide) analogue of adenosine 5'-monophosphate with activity against HIV-1,

Table I Summary of the DHHS and WHO adult and pediatric HIV treatment guideline recommendations ${ }^{9-11}$

2012 DHHS US preferred regimens for antiretroviral-naive adult patients

- Efavirenz/tenofovir/emtricitabine

- Ritonavir-boosted atazanavir + tenofovir/emtricitabine

- Ritonavir-boosted darunavir + tenofovir/emtricitabine

- Raltegravir + tenofovir/emtricitabine

$2010 \mathrm{WHO}$ preferred regimens for antiretroviral-naive adult patients

- Zidovudine + lamivudine + efavirenz

- Zidovudine + lamivudine + nevirapine

- Tenofovir + (lamivudine or emtricitabine) + efavirenz

- Tenofovir + (lamivudine or emtricitabine) + nevirapine

201 I DHHS preferred regimens for 2-NRTI backbone for antiretroviralnaive pediatric patients (for use with additional antiretroviral drugs)

- Abacavir + (lamivudine or emtricitabine) (children age $\geq 3$ months)

- Tenofovir + (lamivudine or emtricitabine) (adolescents age $\geq 12$ years and Tanner stage 4 or 5 only)

- Zidovudine + (lamivudine or emtricitabine)

Abbreviations: DHHS, Department of Health and Human Services; WHO, World Health Organization; NRTI, nucleoside reverse-transcriptase inhibitor.
HIV-2, and hepatitis B. ${ }^{13-15}$ TDF prevents HIV replication by competitively inhibiting the enzyme reverse transcriptase and resulting in DNA chain termination in active and resting lymphoid cells and macrophages. ${ }^{15}$

TDF is formulated as a tablet in strengths of 150, 200, 250 , and $300 \mathrm{mg}$. Recently, a powder formulation has become available for oral administration in children. TDF has been coformulated with several other ARV medications. Truvada (Gilead Sciences) consists of TDF and another NRTI FTC. Atripla (Gilead Sciences and Bristol-Myers Squibb, New York, NY, USA) is a fixed-dose coformulation (FDC) of TDF with FTC and the first-generation non-nucleoside reverse-transcriptase inhibitor (NNRTI) efavirenz (EFV). The newest FDA-approved TDF containing coformulation is Complera (Gilead Sciences), which consists of TDF, FTC, and the second-generation NNRTI rilpivirine (RPV). All coformulations of TDF are especially attractive in clinical practice because they allow for once-daily administration of multiple drugs in a single pill, thus decreasing overall pill burden and creating a better setting for maintaining long-term adherence.

Due to low pill burden, availability in FDC, affordable cost, and storage, TDF is increasingly prescribed as a component of the ARV regimens used for the prevention of HIV transmission, including prevention of mother-to-child transmission and PrEP and postexposure prophylaxis (PEP) in noninfected adolescents and adults. ${ }^{16-18}$ In the Pediatric HIV/AIDS Cohort Study (PHACS), TDF use in HIV-infected women during pregnancy has been reported to have doubled in the last 5 years, with over $40 \%$ of pregnant mothers in the large study cohort receiving TDF. ${ }^{19}$ Recent studies (CAPRISA004, iPrEX, TDF2, Partners for PrEP) have investigated the use of TDF in noninfected adults for PreP of HIV infection primarily through sexual contact. ${ }^{20-24}$ In the CAPRISA004 study, the use of $1 \%$ TFV gel by noninfected women with high risk for HIV exposure reduced risk of HIV acquisition by $39 \% .{ }^{21}$ The studies of oral TDF/FTC (iPREX and TDF2) reported $44 \%$ and $62 \%$ efficacy in preventing HIV transmission, respectively. ${ }^{22,23}$ The study Partners for Prep demonstrated 63\% reduction in HIV transmission in adults using mono-prophylaxis with oral $\mathrm{TDF}^{24}$ Despite some contradicting results on low efficacy of oral TDF/FTC and TFV vaginal gel in two other studies (FEMPrep and VOICE), ${ }^{25,26}$ the evidence from studies demonstrating the efficacy of PreP was considered sufficient by the FDA to approve the TDF-based FDC Truvada (Gilead Sciences) for use as PrEP in noninfected individuals in July 2012. With this new approval, the use of TDF in noninfected adolescents and adults is likely to increase significantly in the near future. 


\section{Pharmacokinetics and toxicity of TDF}

TFV has a long half-life of approximately 17 hours, which allows for convenient once-daily dosing. ${ }^{27,28} \mathrm{TFV}$ is watersoluble, and its oral bioavailability in fasted subjects is approximately $25 \%$. High-fat meals increase bioavailability to $39 \%$ compared to light meals without significant effect. ${ }^{29}$ Unchanged TFV is excreted by the kidneys through a combination of glomerular filtration and active tubular secretion. Renal impairment (creatinine clearance $<50 \mathrm{~mL}$ /minute) requires reduction of TDF dosing with extended dosing intervals. ${ }^{29}$ Since TFV has minimal involvement with hepatic metabolism, no dose adjustment is required for patients with hepatic impairment or coinfection with hepatitis.

TFV is not involved with cytochrome (CYP) P450 metabolism, resulting in fewer drug-drug interactions than other ARV drugs, such as protease inhibitors (PIs). This lack of significant drug-drug interactions is particularly beneficial for HIV-infected patients who may have comorbidities requiring other medications. TDF, however, does have significant interaction with the NRTI didanosine (ddI). Coadministration of TDF and ddI results in increased ddI plasma concentrations and toxicity. DHHS guidelines recommend avoiding coadministration of these ARV drugs. If coadministration is required, ddI dose reduction is recommended with close monitoring for ddI-associated toxicity, such as pancreatitis and peripheral neuropathy.,11

Two important drug-related toxicities - renal and bone toxicity - have been associated with TDF use in children and adults. Rare cases of acute renal failure and Fanconi syndrome (dysfunction of renal tubules resulting in excessive renal loss of glucose, amino acids, and electrolytes) have been described in association with TDF exposure..$^{30-33}$ The cumulative incidence of nephrotoxicity in TDF-containing regimens has been reported between $1 \%$ and $4 \%$, with the rate of Fanconi syndrome between $0.5 \%$ to $2.0 \%{ }^{34}$ Nephrotoxicity most frequently occurs in patients with prior underlying renal abnormalities or those concomitantly exposed to other nephrotoxic agents. In clinical trials and practice, however, serious renal impairment associated with TDF has rarely been reported. ${ }^{35}$ The mechanism for renal toxicity is not well understood, but may involve adverse effects on transporter proteins in the kidney tubules..$^{36}$ Following discontinuation of TDF, associated renal toxicity usually resolves without consequences. ${ }^{37}$ Current drug-manufacturer and treatment recommendations include assessing creatinine clearance prior to initiation and throughout the length of therapy with TDF, avoiding administration of TDF concurrently with other nephrotoxic drugs, and continued monitoring of renal function while on a TDF-containing regimen. ${ }^{9,11,29}$

A decrease in bone mineral density (BMD) has also been observed in HIV-infected adult and pediatric patients treated with TDF-containing regimens, with dual X-ray absorptiometry demonstrating small decreases in spine and hip BMD. The reported decrease in BMD, however, does not appear to be clinically relevant in adult patients, except for the rare cases of osteomalacia reported with TDF use. ${ }^{29,38,39}$ Potential decreases in $\mathrm{BMD}$ are concerning for prepubertal children or adolescents in early stages of pubertal development. Studies have indicated that younger children (Tanner stages 1 and 2 of sexual maturation) may be at higher risk for TDF-associated decreases in BMD than older patients with more advanced stages of sexual development (Tanner stage $\geq 3$ ). ${ }^{11}$

Unlike other NRTIs, such as AZT and d4T, which have been linked to significant mitochondrial damage, TDF has been associated with minimal mitochondrial toxicity. Adverse effects, such as lipoatrophy, lactic acidosis, or peripheral neuropathy, are typically not related to TDF use. ${ }^{40}$ Nevertheless, rare cases of lactic acidosis and severe hepatomegaly with steatosis have been reported in patients treated with TDF, and discontinuation of TDF is recommended in any patient exhibiting clinical or laboratory findings suggestive of these side effects. ${ }^{29}$

\section{Neuropsychiatric complications of HIV and ART}

Neuropsychiatric disease in HIV-infected patients may be caused by HIV infection itself, toxicity from ART, a preexisting underlying neuropsychiatric condition nonrelated to HIV, opportunistic comorbidities resulting from HIV-associated immunosuppression, or a combination of several of these conditions. Primary central nervous system (CNS) lymphoma and progressive multifocal leukoencephalopathy are associated with profound immune suppression. Additionally, opportunistic infections affecting the CNS, such as cryptococcosis and toxoplasmosis, may cause neurological manifestations in severely immunocompromised HIV-infected patients. Moreover, history of substance abuse, head injury, cerebral vascular accident, or aging can also contribute to the development of neuropsychiatric impairment in HIV-infected patients. ${ }^{41}$ Distinguishing the etiology of neuropsychiatric manifestations in HIV-infected patients in order to ensure the most optimal therapeutic approach is crucially important.

\section{Neuropsychiatric effects of HIV}

The direct effect of HIV infection on the CNS has been associated with the development of asymptomatic neurocognitive 
impairment, HIV-associated neurocognitive disorder (HAND), and HIV-associated dementia (HAD), or AIDS dementia complex. HAD is the most severe neuropsychiatric manifestation of HIV. It is a clinical syndrome of severe cognitive, motor, and behavioral dysfunction that usually develops in patients with untreated HIV infection. ${ }^{42}$ HAND is considered to be less severe than HAD, with symptoms including impaired memory, attention, processing speed, and executive functioning; speech, language, visuospatial deficits; and slowing of psychomotor processing and motor speed. $^{43}$

HIV penetrates the CNS soon after infection via infected immune cells, such as $\mathrm{CD}^{+}{ }^{+} \mathrm{T}$ lymphocytes, dendritic cells, monocytes, and macrophages. As a major target of HIV, the CNS continues to be affected by the virus throughout the course of the disease. ${ }^{44}$ Subtype-D HIV has been implicated in being more neurovirulent than other subtypes in adults. ${ }^{45}$ Cognitive impairment frequently develops secondary to chronic inflammation, resulting in neurodegeneration. ${ }^{46}$ The level of HIV viremia (HIV RNA) in cerebrospinal fluid (CSF) has been shown to correlate with severity of HIV-associated dementia and neuropsychological test performance. Elevated HIV RNA in CSF can predict the development of subsequent neurocognitive impairments. ${ }^{47-51}$ Following the introduction and effective application of ART in clinical settings, a profound reduction in the incidence and severity of HAD, as well as major CNS opportunistic infections, has been observed. ${ }^{43}$ The dramatic decrease in incidence of HAD from $>50 \%$ to $2 \%-8 \%$ has been suggested to be mediated by suppression of HIV replication and reduction in circulating lipopolysaccharide, both preventing compromise of the blood-brain barrier and migration of HIV-infected monocytes from systemic circulation into the brain. ${ }^{52,53}$ A multicenter study of HIV-infected older children (7-16 years of age) in the US and Puerto Rico demonstrated that neurocognitive deficits acquired in early childhood were reversible with consistent use of ART in pediatric patients with HIV infection. ${ }^{54}$ As a component of cART therapy, TFV has contributed to the decrease in severe neuropsychiatric manifestations of HIV disease.

Although clear evidence supports the success of cART in prevention of the severe neuropsychiatric disease associated with HIV, the prevalence of minor HIV-associated cognitive impairment appears not to be affected by cART and has been noted to be on the rise in recent years. ${ }^{55,56}$ This effect can be partially due to improved survival and longevity of the patients with HIV infection with modern, highly effective cART. Cohort studies have described a prevalence of demonstrable cognitive impairments in 30\%-60\% of HIV-infected adults. ${ }^{44}$ Not surprisingly, the history of low nadir in $\mathrm{CD}^{+}$cell count below 50 cells $/ \mathrm{mm}^{3}$ was linked to a greater risk of neurocognitive impairment. ${ }^{56}$ Similar to adults, children with history of severe immunosuppression were also more likely to have residual neurocognitive deficits. ${ }^{56}$ The constant presence of low levels of HIV in the CNS along with the associated immune activation and ongoing inflammation may be related to more subtle forms of brain injury, which has become a focus of clinical attention and research in the cART era. ${ }^{44}$

HAND and neurocognitive impairment continue to be diagnosed even among HIV-infected patients with immune recovery on cART..$^{53,57,58}$ The presence of HAND in patients effectively treated with ARVs may be caused by limited penetration and distribution of ARV drugs in the CNS. ${ }^{46}$ The normal function of the blood-brain and blood-CSF barriers is to shield the brain from harmful, toxic substances and provide a precisely regulated environment in the CNS. The CNS acts as a sanctuary site for HIV, and HIV replication can persist despite virologic suppression in blood and other tissues. ${ }^{59}$ This ongoing low-level viral replication within the CNS may lead to neuronal damage, resulting in long-term cognitive impairment.

Minor HIV-associated cognitive impairment may also be caused by the insurgence of drug-resistant HIV in the CNS while on cART. ${ }^{60}$ By limiting CNS penetration of ARV drugs, the blood-brain/blood-CSF barrier potentially decreases CNS toxicity of ARV medications, but also contributes to continued presence of HIV in the CNS despite an undetectable HIV RNA viral load in the peripheral blood stream. Hindering the penetration of ARV drugs into the CNS can therefore result in subtherapeutic ARV drug concentrations, resulting in the development of drug resistance and ongoing replication of the HIV within CNS. Ultimately, this may contribute to treatment failure and persistent neurocognitive deficits, even in the presence of effective cART. ${ }^{46}$

\section{Neuropsychiatric effects of ART}

Continued presence of minor cognitive impairment in HIVinfected patients on ART may be caused by the toxicity of ARV drugs, especially those with higher CNS penetration. ART-related CNS toxicity may be due to direct ARV drug CNS toxicity and/or CNS immunopathology related to immune restoration in the presence of ART. ${ }^{60}$ Efforts have been made to classify ARV drugs according to their ability to penetrate the CNS. In 2008, Letendre et al devised and validated a method to quantify the penetration of ARVs into 
the CNS by giving each ARV drug a CNS penetration effectiveness (CPE) score. ${ }^{61}$ Currently available information about ARV drug characteristics, measured CSF concentrations, and effectiveness in the CNS were used to rank ARV drugs relative to one another with regard to their ability to penetrate the CNS. The authors revised the CPE ranking in 2010 to include ARVs from newer classes of drugs (Table 2). ${ }^{46,61} \mathrm{ARV}$ regimens with estimated good CNS penetration are generally more effective than regimens with worse CNS penetration in controlling CSF viral replication, and may contribute to the more significant restoration and preservation of neurocognitive function. Smurzynski et al found that in patients using ART regimens containing more than three ARV drugs, the use of high versus low CPE scoring regimens was correlated with better scores on neurocognitive testing. This relationship, however, was not reported in patients using ART with three or less ARV drugs. ${ }^{62}$

Better CNS-penetrating ART provides better control of viral replication within the CNS; however, HIV suppression below the levels of detection does not guarantee full cognitive recovery. ${ }^{46}$ Interestingly, a study by Marra et al suggested that ARV drugs with good CNS penetration were associated with worse neurocognitive performance. ${ }^{63}$ In this study, ARV regimens with $\mathrm{CPE} \geq 2$, indicating good CNS penetration, were compared to regimens with $\mathrm{CPE}<2$. The use of a regimen with $\mathrm{CPE} \geq 2$ was significantly associated

Table 2 CPE scores and selected common CNS adverse events associated with ARV medications (as cited in DHHS guidelines for the use of antiretroviral agents in HIV-I-infected adults and adolescents and guidelines for the use of antiretroviral agents in pediatric HIV infection) $)^{9,11,46}$

\begin{tabular}{|c|c|c|c|c|c|c|c|}
\hline ARV drug & CPE score* & Headache & Dizziness & Asthenia & Insomnia & Depression & Other CNS^ \\
\hline \multicolumn{8}{|c|}{ Nucleoside (nucleotide) reverse-transcriptase inhibitors } \\
\hline Zidovudine & 4 & $\mathrm{x}$ & & $x$ & $x$ & & \\
\hline Abacavir & 3 & $x$ & & & & & \\
\hline Didanosine & 2 & & & & & & \\
\hline Emtricitabine & 3 & $x$ & & & & & \\
\hline Lamivudine & 2 & $x$ & & & & & \\
\hline Stavudine & 2 & $x$ & & & & & \\
\hline Tenofovir & I & $\mathrm{x}$ & & $x$ & & & \\
\hline \multicolumn{8}{|c|}{ Nonnucleoside reverse-transcriptase inhibitors } \\
\hline Efavirenz & 3 & $\mathrm{x}$ & $x$ & & $x$ & $x$ & $x$ \\
\hline Etravirine & 2 & & & & & & \\
\hline Nevirapine & 4 & & & & & & \\
\hline Rilpivirine & 2 & $x$ & & & $x$ & $x$ & \\
\hline \multicolumn{8}{|l|}{ Protease inhibitors } \\
\hline Atazanavir & & $x$ & $x$ & & $x$ & $x$ & \\
\hline Atazanavir/ritonavir & 2 & & & & & & \\
\hline Darunavir & & $x$ & & & & & \\
\hline Darunavir/ritonavir & 3 & & & & & & \\
\hline Fosamprenavir & 2 & $x$ & & & & & \\
\hline Fosamprenavir/ritonavir & 3 & & & & & & \\
\hline Indinavir & 3 & $x$ & $x$ & $x$ & & & \\
\hline Indinavir/ritonavir & 4 & & & & & & \\
\hline Lopinavir/ritonavir ${ }^{\ddagger}$ & 3 & $x$ & & $x$ & & & \\
\hline Nelfinavir & 1 & & & $x$ & & & \\
\hline Ritonavir & 1 & $x$ & & $x$ & & & \\
\hline Tipranavir $^{\dagger}$ & & $x$ & & & & & \\
\hline Tipranavir/ritonavir & I & & & & & & \\
\hline Saquinavir & I & $x$ & & & & & \\
\hline Saquinavir/ritonavir & I & & & & & & \\
\hline \multicolumn{8}{|l|}{ Entry inhibitors } \\
\hline Enfuvirtide & I & & & & & & \\
\hline Maraviroc & 3 & & $x$ & & & & \\
\hline \multicolumn{8}{|l|}{ Integrase inhibitor } \\
\hline Raltegravir & 3 & $x$ & $x$ & & $x$ & & \\
\hline
\end{tabular}

Notes: ^Other CNS symptoms include somnolence, abnormal dreams, impaired concentration, agitation, psychosis, abnormal thinking, hallucinations, euphoria, seizures; trare cases of intracranial hemorrhage reported in adults; łglobal CNS depression reported in premature infants. into the CNS $(4=$ highest penetration, $3=$ high, $2=$ intermediate, I = low); *larger numbers reflect estimates of better penetration.

Abbreviations: ARV, antiretroviral; CNS, central nervous system; CPE, CNS penetration effectiveness. 
with worse Z-scores on a short-battery neuropsychological test despite better control of CSF viral replication with ARV regimens with good CSF penetration. The authors concluded that ARV regimens with better CSF penetration may not lead to improved neurocognitive performance. It is important to recognize that the interpretation of study results was limited due to the small number of subjects, with only 75 subjects participating in the short-battery neuropsychological testing. Another study of HIV-infected adults compared the overall neurological and neuropsychological effects of ARV regimens. ${ }^{64}$ In this study, by Robertson et al, subjects were randomized to three treatment arms: A (lamivudine [3TC], AZT, plus EFV), B (FTC, ddI, plus atazanavir [ATV]) and C (FTC, TDF, plus EFV). Standardized neurological and neuropsychological examinations were administered every 24 weeks during the study period of 4 years. Each study arm was assigned a CPE score according to Letendre's ranking system. The total CPE score for arms A, B, and C, were 9, 7, and 7, respectively, with a higher score indicating higher CNS penetration of the overall ART regimen. The study detected no significant differences in neurological and neuropsychological outcomes between the randomized ART combinations. Authors concluded that regimens with higher CPE scores did not correlate with additional neurological or neuropsychological benefit. Overall, however, significant improvement in neurological and neuropsychological outcomes did occur over time after initiation of ART, regardless of ARV drug combination.

Although overall rates of treatment-limiting CNS adverse events of ART are low, especially with newer ARV drugs, cognizance of such events is important, since they can directly affect adherence to ART. ${ }^{9}$ Drug-induced CNS adverse reactions attributable to individual ARV drugs can result in a spectrum of symptoms, ranging from sleep disturbance to cognitive impairment and psychosis. ${ }^{65}$ Complex drug interactions between ARV drugs could also contribute to CNS toxicity. ${ }^{66}$ Published case reports and clinical studies of ARV-associated CNS toxicity most commonly report causative relationships between neuropsychiatric complications of ART and NRTIs (AZT and abacavir [ABC]), NNRTIs (EFV and RPV) and several PIs. ${ }^{47}$

While neuropsychiatric side effects may occur with almost all ARV medications, the majority of them are selflimited and do not result in discontinuation of medication. In a study that reviewed reasons for discontinuation of ART, adverse events accounted for $24 \%(n=84)$ of reasons for discontinuing therapy. ${ }^{67}$ Of all adverse events $(n=84)$, CNS complaints were cited in significantly smaller proportion
$(10 \% ; n=8)$, compared to the gastrointestinal abnormalities observed in $44 \%(n=37)$ of patients. Among the CNS complaints, headache, insomnia, and dizziness were responsible for $7 \%, 7 \%$, and $5 \%$ of all adverse events, respectively. ${ }^{67}$

Headache is the most frequently cited adverse event for NRTIs such as AZT, abacavir, FTC, 3TC, d4T, and TDF for children and adults., ${ }^{911}$ Typically, the headache is transient, mild to moderate in severity, and can be treated symptomatically. AZT has also been associated with the side effects of insomnia and asthenia..$^{9,11}$

Headache has also been implicated as a common drug toxicity for certain PIs, including ATV, darunavir, fosamprenavir, indinavir (IDV), lopinavir/ritonavir (LPV/RTV) and tipranivir. Additionally, IDV, LPV/RTV, and RTV have been associated with asthenia, while ATV and IDV have also been associated with dizziness. ${ }^{9,11}$ As with NRTIs, these side effects are often self-limited and only mild to moderate in severity, usually not requiring a switch in ART. Notably, tipranivir has been linked with rare cases of intracranial hemorrhage and should be used cautiously in patients with prior history of intracranial lesions or bleeding disorder. ${ }^{9}$ The propylene glycol and alcohol components of the LPV/RTV oral solution may also contribute to global CNS depression reported in neonates and preterm infants and limits the use of this combination PI in premature and young infants to those with postmenstrual age of $>42$ weeks and a postnatal age of $>14$ days. ${ }^{11}$ LPV/ RTV-associated CNS depression typically resolves within 1-5 days of discontinuation of the drug. ${ }^{9,11}$

Neuropsychiatric side effects of the newer classes of ARV medications, such as the entry inhibitor maraviroc, include dizziness and postural hypotension, while the integrase inhibitor raltegravir has been associated with headaches and dizziness. ${ }^{9,11}$ Table 2 summarizes selected common CNS adverse events for ARV drugs as cited by DHHS adult and pediatric guidelines for use of ARV agents., ${ }^{9,10}$

A high incidence of the neuropsychiatric effects, ranging from $40 \%$ to $70 \%$, has been reported in association with NNRTI EFV. ${ }^{68,69}$ EFV crosses the blood-brain barrier well, and CSF-to-plasma ratios have been found to range between $0.65 \%$ and $1.19 \% .^{70}$ Neuropsychiatric manifestations most commonly associated with EFV use include dizziness, confusion, lethargy, impaired concentration, amnesia, hallucinations, abnormal dreams, insomnia, sleep disorders, depression, and irritability. ${ }^{71,72}$ The CNS symptoms usually appear in the first weeks of therapy and abate within several weeks. ${ }^{73-77}$ While the exact mechanism responsible for these neuropsychiatric effects by EFV has not been established, some studies suggest that the CNS side effects are related to 
the higher level of plasma EFV exposure $>4000 \mathrm{ng} / \mathrm{mL}^{78}$ Since EFV is the substrate to the CYP450 2B6 metabolic pathway, the polymorphisms in CYP2B6 (particularly 516 T > G) has been reported to increase the EFV plasma exposure and the severity of EFV-associated neurotoxicity. ${ }^{79}$

A second-generation NNRTI RPV has also been associated with CNS adverse events, including depression, insomnia, and headache. From safety and efficacy studies comparing RPV and EFV, of 686 subjects on RPV, the most common treatment-related adverse events ( $\geq$ grade 2 ) included dizziness $(1 \%, n=7)$, abnormal dreams/nightmares $(1 \%, n=4)$, headache $(2 \%, n=9)$, and insomnia $(2 \%, n=12) .{ }^{80}$ When considering all grades of RPV-related adverse events, any form of neurologic adverse event was reported in 17\% $(n=117)$ of the subjects, with $8 \%(n=55)$ complaining of dizziness, while any form of psychiatric adverse event was reported in $15 \%(n=102)$ of subjects, with $8 \%(n=56)$ complaining of abnormal dreams/nightmares. The frequency of dizziness and abnormal dreams/nightmares for RPV was significantly lower than that reported in association with $\mathrm{EFV}$, whereas the incidence of depression was similarly low for both groups.

\section{Neuropsychiatric effects of TDF}

TFV belongs to the class of ARV drugs with low penetration into the CNS. Animal data indicate that TFV is transported across the blood-CSF barrier but not the blood-brain barrier, due to physicochemical properties of the drug. Its high polarity and low lipid solubility reduce its ability to readily undergo passive diffusion to cross the blood-brain barrier. ${ }^{81}$ The penetration of TFV into the brain, therefore, relies on active transport systems to cross the blood-brain/bloodCSF barrier. These transporters are responsible for both efflux and influx of TFV into the CNS. ${ }^{82}$ Concentrations of TFV in the CSF have been found to be only $5 \%$ of plasma concentrations. ${ }^{83}$

With limited penetration into the CNS, TFV is unlikely to produce direct adverse effects on the CNS. During phase II and III clinical trials, TDF exhibited limited neuropsychiatric effects. In the Gilead Sciences study GS-99-903, a double-blind comparative controlled trial designed to evaluate the safety of TDF in adults, the most common neuropsychiatric adverse reactions attributable to the study drug were headache and dizziness. ${ }^{38}$ Overall, from three large controlled clinical trials, the most common TDF-associated neuropsychiatric adverse reactions with incidence $\geq 10 \%$ and grades 2-4 included headache, depression, and asthenia. ${ }^{29}$ In an equivalency trial designed to assess efficacy and safety of TDF, cART consisting of TDF, 3TC, and EFV was compared to a regimen containing $\mathrm{d} 4 \mathrm{~T}, 3 \mathrm{TC}$, and EFV. Overall frequency of grade 2-4 neuropsychiatric adverse events including asthenia $(6 \%-7 \%)$, depression $(10 \%-11 \%)$, and anxiety $(6 \%)$ were similar between the two groups throughout 144 weeks of follow-up. A smaller proportion of patients using the TDF/3TC/EFV regimen reported headache (14\% versus $17 \%$ ), insomnia ( $5 \%$ versus $8 \%$ ), dizziness ( $3 \%$ versus $6 \%$ ), and peripheral neuropathy ( $1 \%$ versus $5 \%$ ) compared to those taking d4T/3TC/EFV. ${ }^{29,79}$ In an open-label noninferiority study comparing two ARV regimens TDF/FTC/EFV with AZT/3TC/EFV, patients receiving a TDF-containing ART reported similar frequency of neuropsychiatric adverse events in grades $2-4$, such as headache (5\%-6\%), dizziness (7\%-8\%), insomnia (5\%-7\%), and depression (7\%-9\%) as those on a regimen without TDF. ${ }^{29,83}$

A case series described nine episodes of neuropsychiatric intolerance occurring early after a switch from an ART regimen without TDF to a TDF-containing regimen. ${ }^{84}$ Moderate to severe neuropsychiatric adverse events were noted almost immediately after TDF initiation. CNS symptoms included nightmare $(n=4)$, insomnia $(n=3)$, dizziness $(n=4)$, tinnitus $(n=1)$, nausea $(n=1)$, and irritability $(n=1)$. CNS symptoms occurring during the 24 months after initiation of TDF included nightmares $(n=4)$ and insomnia $(n=2)$. Due to these side effects, five out of the nine patients switched ARV regimen (discontinuing TDF), and one discontinued ARV therapy altogether. CNS symptoms improved in all six who changed or discontinued regimens. In the three patients who did not switch regimens, one had spontaneous resolution of symptoms within 2 weeks, and two continued to experience sleeping disorders. The mechanism of the emerging CNS adverse effects after the introduction of TDF to an EFV-containing regimen remains unclear. It was hypothesized that the neuropsychiatric effects may have been the result of an unexplained interaction between EFV and TDF that resulted in increased levels of EFV, likely causing the neuropsychiatric side effects. ${ }^{84}$ The possibility of PK interaction between TDF and EFV has been questioned, especially among patients with polymorphism of CYP2B6 who are slow EFV metabolizers. ${ }^{85}$ The study, evaluating EFV and TDF exposure, reported similar EFV area under the plasma vs time curve concentrations (AUC) among the adult patients, except for the significantly higher exposure among patients with CYP2B6 polymorphism and patients receiving concomitant TFV $(\mathrm{n}=5 ; \mathrm{AUC}=353,031 \mathrm{ng} * \mathrm{~h} / \mathrm{mL})$ compared to those not receiving TFV $(\mathrm{n}=18 ; \mathrm{AUC}=180,689$ $\mathrm{ng}{ }^{*} \mathrm{~h} / \mathrm{mL}$ ). Typically, however, the concomitant administration of TDF and EFV has not been associated with significant $\mathrm{PK} /$ pharmacodynamic interactions. ${ }^{86}$ 


\section{Conclusion}

As the armamentarium of ARV drugs continues to grow, understanding the potential benefits and risks of each ARV agent is essential in designing the most optimal regimen for individual patients. TDF, coformulated in multiple FDCs, is widely recommended for treatment and prophylaxis of HIV infection due to its potency, safety, tolerability, and convenient dosing. The potency of TDF has been instrumental in successfully suppressing viral replication and decreasing incidence of severe neuropsychiatric disorders associated with advanced HIV infection. The low CNS penetration by TFV is likely responsible for the minimal spectrum of neuropsychiatric adverse effects associated with the drug. The same low CNS penetration of TFV, however, may limit its ability to independently reduce HIV viral load in the CNS, potentially contributing to persistent low-level HIV viremia, leading to mild neuropsychiatric impairments and/or development of drug resistance. Combining TDF with ARV drugs with high CNS penetration may be strategically beneficial for the long-term management of HIV-infected patients. In patients on TDF-containing regimens, early recognition of neuropsychiatric disorders is essential to achieve a high quality of life and most beneficial long-term outcome of HIV infection.

More clinical studies evaluating the long-term neuropsychological, neurocognitive, and neurodevelopmental effects of ARV regimens, particularly among HIV-infected children and elderly patients, are needed. Specifically, future studies should evaluate the relationship between particular ARV regimen CPE scores, CSF viral replication, and overall neurological outcome to allow clinicians to estimate the benefits and risks of high CSF penetrating ART. Eventually, this research could provide valuable insight for improving the selection of ART to ensure most optimal long-term neurological outcomes for HIV-infected patients.

\section{Acknowledgment}

N Rakhmanina is supported by Department of Health and Human Services, NIH PHS grant NICHD K23 HD060452.

\section{Disclosure}

The authors have no conflicts of interest to declare.

\section{References}

1. Palella FL Jr, Delaney KM, Moorman AC, et al. Declining morbidity and mortality among patients with advanced human immunodeficiency virus infection. HIV Outpatient Study Investigators. $N$ Engl J Med. 1998;338(13):853-860.

2. Berrey MM, Schacker T, Collier AC, et al. Treatment of primary human immunodeficiency virus type 1 infection with potent antiretroviral therapy reduces frequency of rapid progression to AIDS. J Infect Dis. 2001;183(10):1466-1475.
3. Gallant JE, Staszewski S, Pozniak AL, et al. Efficacy and safety of tenofovir DF vs stavudine in combination therapy in antiretroviral-naïve patients: a 3-year randomized trial. JAMA. 2004;292(2):191-201.

4. Negredo E, Moltó J, Muñoz-Moreno JA, et al. Safety and efficacy of once-daily didanosine, tenofovir and nevirapine as a simplification antiretroviral approach. Antivir Ther. 2004;9(3):335-342.

5. Louie M, Hogan C, Hurley A, et al. Determining the antiviral activity of tenofovir disoproxil fumarate in treatment-naïve chronically HIV1-infected individuals. AIDS. 2003;17(8):1151-1156.

6. Kearney BP, Abadi J, Rosenberg M, et al. Pharmacokinetics (PK) of tenofovir DF (TDF) oral suspension in HIV-1 infected children between 2 and 8 years of age. Paper presented at the 11th Conference on Retroviruses and Opportunistic Infections; February 8-11, 2004; San Francisco, CA, USA.

7. Ramos JT, Gonzalez Tome MI, Rojo P, Fernandez Ibieta M, Vargas K, Garcia-Pinal L. Experience with tenofovir disoproxil fumarate (TDF) in heavily pretreated HIV-infected children Paper presented at the 15th International AIDS Conference; July 11-16, 2004; Bangkok, Thailand.

8. Hazra R, Gani RI, Maldarelli F, et al. Tenofovir disoproxil fumarate and an optimized background regimen of antiretroviral agents as salvage therapy for pediatric HIV infection. Pediatrics. 2005;116(6):e846-e854.

9. Panel on Antiretroviral Guidelines for Adults and Adolescents. Guidelines for the use of antiretroviral agents in HIV-1-infected adults and adolescents. Updated January 12, 2012. Available from: http:// aidsinfo.nih.gov/contentfiles/lvguidelines/adultandadolescentgl.pdf. Accessed December 25, 2012.

10. World Health Organization. Antiretroviral Therapy for HIV Infection in Adults and Adolescents: Recommendations for a Public Health Approach. Revised. Geneva: WHO; 2010. Available from: http://www. who.int/hiv/pub/arv/adult2010/en/index.html. Accessed October 15, 2012.

11. Panel on Antiretroviral Therapy and Medical Management of HIVInfected Children. Guidelines for the use of antiretroviral agents in pediatric HIV infection. Updated August 11, 2011. Available from http://aidsinfo.nih.gov/Contentfiles/PediatricGuidelines.pdf. Accessed October 15, 2012.

12. Cohen MS, McCauley M, Gamble TR. HIV treatment as prevention and HPTN 052. Curr Opin HIV AIDS. 2012;7(2):99-105.

13. Balzarini J, Holy A, Jindrich J, et al. Differential antiherpesvirus and antiretrovirus effects of the (S) and (R) enantiomers of acyclic nucleoside phosphonates: potent and selective in vitro and in vivo, antiretrovirus activities of (R)-9- (2-phosphonomethoxypropyl)-2,6diaminopurine. Antimicrob Agents Chemother. 1993;37(2):332-338.

14. Robbins BL, Srinivas RV, Kim C, Bischofberger N, Fridland A. Anti-human immunodeficiency virus activity and cellular metabolism of a potential prodrug of the acyclic nucleoside phosphonate 9-R-(2-phosphonomethoxypropyl)adenine (PMPA), bis(isopropyloxymethylcarbonyl) PMPA. Antimicrob Agents Chemother. 1998;42(3):612-617.

15. Mulato AS, Cherrington JM. Anti-HIV activity of adefovir (PMEA) and PMPA in combination with antiretroviral compounds: in vitro analyses. Antiviral Res. 1997;36(2):91-97.

16. Centers for Disease Control and Prevention (CDC). Interim guidance: pre-exposure prophylxais for the prevention of HIV in men who have sex with men. MMWR Morb Mortal Wkly Rep. 2011;60(3):65-68.

17. Centers for Disease Control and Prevention (CDC). Updated US public health service guidelines for the management of occupational exposures to HIV and recommendations for postexposure prophylaxis. $M M W R$ Morb Mortal Wkly Rep. 2005;54(RR-9):1-17.

18. Smith DK, Grohskopf LA, Black RJ, et al. Antiretroviral postexposure prohylaxis after sexual, injection-drug use, or other nonoccupational exposure to HIV in the United States: recommendations from the US Department of Health and Human Services. MMWR Recomm Rep. 2005;54(RR-2):1-20.

19. Siberry G, Williams P, Mendez H, et al. Safety of tenofovir use during pregnancy: early growth outcomes in HIV-exposed uninfected infants. AIDS. 2012;26(9):1151-1159. 
20. van der Straten A, Van Damme L, Haberer J, Bangsberg D. Unraveling the divergent results of PrEP trials for HIV prevention. AIDS. 2012;26(7):F13-F19.

21. Abdool Karim Q, Abdool Karim SS, Frohlich JA, et al. Effectiveness and safety of tenofovir gel, an antiretroviral microbicide, for the prevention of HIV infection in women. Science. 2010;329(5996):1168-1174.

22. Grant RM, Lama JR, Anderson PL, et al. Preexposure chemoprophylaxis for HIV prevention in men who have sex with men. $N$ Engl $J$ Med. 2010;363(27):2587-2599.

23. Thigpen M, Debabetswe P, Smith D, et al. Daily oral antiretroviral use for the prevention of HIV infection in heterosexually active young adults in Botswana: results from the TDF2 study. Paper presented at the 6th International AIDS Society Conference on HIV Pathogenesis, Treatment and Prevention; July 17-20, 2011; Rome, Italy.

24. Baeten J, Celum C. Antiretroviral pre-exposure prophylaxis for HIV-1 prevention among heterosexual African men and women: the partners PrEP Study. Paper presented at the 6th International AIDS Society Conference on HIV Pathogenesis, Treatment and Prevention; July 17-20, 2011; Rome, Italy.

25. Family Health International. FHI statement on the FEM-PrEP HIV prevention study: FHI to initiate orderly closure of FEM-PrEP. 2011. Available from: http://www.mtnstopshiv.org/sites/default/files/ attachments/FEM-PrEP\%20Statement\%20FINAL1.pdf. Accessed September 1, 2012.

26. Family Health International. VOICE HIV prevention trial continues, but researchers suspend oral tenofovir arm because of futility. Available from: http://www.fhi360.org/en/AboutFHI/Media/Releases/ res_VOICE.htm. Accessed September 1, 2012.

27. Grim SA, Romanelli F. Tenofovir disoproxil fumarate. Ann Pharmacother. 2003;37(6):849-859

28. Gallant JE, Deresinski S. Tenofovir disoproxil fumarate. Clin Infect Dis. 2003;37(7):944-950.zzzz

29. Viread [package insert]. 2012. Gilead Sciences, Foster City, CA, USA. Available from: http://www.gilead.com/pdf/viread_pi.pdf. Accessed September 5, 2012.

30. Barrios A, García-Benayas T, González-Lahoz J, et al. Tenofovir-related nephrotoxicity in HIV-infected patients. AIDS. 2004;18(6):960-963.

31. James CW, Steinhaus MC, Szabo S, et al. Tenofovir-related nephrotoxicity: case report and review of the literature. Pharmacotherapy. 2004;24(3):415-418.

32. Murphy MD, O’Hearn M, Chou S. Fatal lactic acidosis and acute renal failure after addition of tenofovir to an antiretroviral regimen containing didanosine. Clin Infect Dis. 2003;36(8):1082-1085.

33. Schaaf B, Aries SP, Kramme E, et al. Acute renal failure associated with tenofovir treatment in a patient with acquired immunodeficiency syndrome. Clin Infect Dis. 2003;37(3):c41-c43.

34. Sax PE, Gallant JE, Klotman PE. Renal safety of tenofovir disoproxil fumarate. AIDS Read. 2007;17(2):90-92, 99-104, C3.

35. Scott JD, Wolfe PR, Bolan RK, Guyer W. Serious renal impairment occurs rarely with use of tenofovir DF. HIV Clin Trials. 2006;7(2): 55-58.

36. Rodríguez-Nóvoa S, Labarga P, Soriano V, et al. Predictors of kidney tubular dysfunction in $\mathrm{HIV}$-infected patients treated with tenofovir: a pharmacogenetic study. Clin Infect Dis. 2009;48(11):e108-e116.

37. Woodward CLN, Hall AM, Williams IG, et al. Tenofovir-associated renal and bone toxicity. HIV Med. 2009;10(8):482-487.

38. Cassetti I, Madruga JV, Suleiman JM, et al. The safety and efficacy of tenofovir DF in combination with lamivudine and efavirenz through 6 years in antiretroviral-naïve HIV-1-infected patients. HIV Clin Trials 2007;8(3):164-172.

39. Gafni RI, Hazra R, Reynolds JC, et al. Tenofovir disproxil fumarate and an optimized background regimen of antiretroviral agents as salvage therapy: impact on bone mineral density in HIV-infected children. Pediatrics. 2006;118:e711-e718.

40. Birkus G, Hitchcock MJM, Cihlar T. Assessment of mitochondrial toxicity in human cells treated with tenofovir. Antimicrob Agents Chemother. 2002;46:716-723.
41. McArthur JC, Sacktor N, Selnes OA. Human immunodeficiency virusassociated dementia. Semin Neurol. 1999;19(2):129-150.

42. Antinori A, Arendt G, Becker JT, et al. Updated research nosology for HIV-associated neurocognitive disorders. Neurology. 2007;69(18): 1789-1799.

43. Dennis BC, Houff SA, Han DY, Schmitt FA. Development of neurocognitive disorders in HIV/AIDS. Neurobehav HIV Med. 2011; 2011(3):9-18.

44. Varatharajan L, Thomas SA. The transport of anti-HIV drugs across blood-CNS interfaces: summary of current knowledge and recommendations for further research. Antiviral Res. 2009;82(2):A99-A109.

45. Sacktor N, Nakasujja, Skolasky R, et al. HIV subtype D is associated with dementia, compared with subtype $\mathrm{A}$, in immunosuppressed individuals at risk of cognitive impairment in Kampala, Uganda. Clin Infect Dis. 2009;49(5):780-786.

46. Letendre S, Ellis R, Ances B, McCutchan J. Neurologic complications of HIV disease and their treatment. Top HIV Med. 2010;18(2):45-55.

47. Cespedes M, Aberg J. Neuropsychiatric complications of antiretroviral therapy. Drug Saf. 2006;29(10):865-874.

48. Brew BJ, Pemberton L, Cunningham P, Law MG. Levels of human immunodeficiency virus type 1 RNA in cerebrospinal fluid correlate with AIDS dementia stage. J Infect Dis. 1997;175(4):963-966.

49. McArthur JC, McClemon DR, Cronin MF, et al. Relationship between human immunodeficiency virus-associated dementia and viral load in cerebrospinal fluid and brain. Ann Neurol. 1997;42(5):689-698.

50. Ellis RJ, Hsia K, Spector SA, et al. Cerebrospinal fluid human immunodeficiency virus type 1 RNA levels are elevated in neurocognitively impaired individuals with acquired immunodeficiency syndrome. Ann Neurol. 1997;42:679-688.

51. Ellis RJ, Moore DJ, Childers ME, et al. Progression to neuropsychological impairment in human immunodeficiency virus infection predicted by elevated cerebrospinal fluid levels of human immunodeficiency virus RNA. Arch Neurol. 2002;59(6):923-928.

52. Wang H, Sun J, Goldstein H. Human immunodeficiency virus type 1 infection increases the in vivo capacity of peripheral monocytes to cross the blood-brain barrier into the brain and the in vivo sensitivity of the blood-brain barrier to disruption by lipopolysaccharide. J Virol. 2008;82(15):7591-7600.

53. Heaton RK, Clifford DB, Franklin DR Jr. HIV-associated neurocognitive disorders persist in the era of potent antiretroviral therapy: CHARTER Study. Neurology. 2010;75(23):2087-2096.

54. Smith R, Chernoff M, Rutstein R, et al. The long-term impact of HIV disease severity on cognitive and adaptive functioning during childhood and adolescence. Paper presented at the 16th Conference on Retroviruses and Opportunistic Infections; February 8-11, 2009; Montreal, Canada.

55. Simioni S, Cavassini M, Annoni JM, et al. Cognitive dysfunction in HIV patients despite long-standing suppression of viremia. AIDS. 2010;24(9):1243-1250.

56. Ellis R, Heaton R, Letendre S, et al. Higher CD4 nadir is associated with reduced rates of HIV-associated neurocognitive disorders in the CHARTER study: potential implications for early treatment initiation. Paper presented at the 16th Conference on Retroviruses and Opportunistic Infections; February 8-11, 2009; Montreal, Canada.

57. Tozzi V, Balestra P, Bellagamba R, et al. Persistence of neuropsychologic deficits despite long-term highly active antiretroviral therapy in patients with HIV-related neurocognitive impairment: prevalence and risk factors. J Acquir Immune Defic Syndr. 2007;45(2):174-182.

58. Dawes S, Suarez P, Casey CY, et al. Variable patterns of neuropsychological performance in HIV-1 infection. J Clin Exp Neuropsychol. 2008;30(6):613-626.

59. Blankson JN, Persaud D, Siliciano RF. The challenge of viral reservoirs in HIV-1 infection. Annu Rev Med. 2002;53(1):557-593.

60. Price RW, Spudich S. Antiretroviral therapy and central nervous system HIV type 1 infection. J Infect Dis. 2008;197(Suppl 3):S294-S306.

61. Letendre S, Marquie-Beck J, Capparelli E, et al. Validation of the CNS penetration-effectiveness rank for quantifying antiretroviral penetration into the central nervous system. Arch Neurol. 2008;65(1):65-70. 
62. Smurzynski M, Wu K, Letendre S, et al. Effects of central nervous system antiretroviral penetration on cognitive functioning in the ALLRT cohort. AIDS. 2011;25:357-365.

63. Marra CM, Zhao Y, Clifford DB, et al. Impact of combination antiretroviral therapy on cerebrospinal fluid HIV RNA and neurocognitive performance. AIDS. 2009;23(11):1359-1366.

64. Robertson K, Jiang H, Kumwenda J, et al. Improved neuropsychological and neurological functioning across three antiretroviral regimens in diverse resource-limited settings: AIDS Clinical Trials Group study A5199, the International Neurological Study. Clin Infect Dis. 2012; 55(6):868-876.

65. Blanch J, Martinez E, Rousaud A, et al. Preliminary data of a prospective study on neuropsychiatric side effects after initiation of efavirenz. J Acquir Immune Defic Syndr. 2001;27(4):336-343.

66. Cysique LA, Maruff P, Brew BJ. Antiretroviral therapy in HIV infection: are neurologically active drugs important? Arch Neurol. 2004;61(11):1699-1704.

67. O’Brien ME, Clark RA, Besch CL, Myers L, Kissinger P. Patterns and correlates of discontinuation of the initial HAART regimen in an urban outpatient cohort. J Acquir Immune Defic Syndr. 2003;34(4): $407-414$.

68. Gazzard B, Balkin A, Hill A. Analysis of neuropsychiatric adverse events during clinical trials of efavirenz in antiretroviral-naïve patients: a systematic review. AIDS Rev. 2010;12(2):67-75.

69. Cavalcante GIT, Capistrano VL, Cavalcante FS, et al. Implications of efavirenz for neuropsychiatry: a review. Int J Neurosci. 2010;120(12): 739-745.

70. Tashima KT, Caliendo AM, Ahmad M, et al. Cerebrospinal fluid human immunodeficiency virus type 1 (HIV-1) suppression and efavirenz drug concentrations in HIV-1-infected patients receiving combination therapy. J Infect Dis. 1999;180(3):862-864.

71. Jena A, Sachdeva RK, Sharma A, Wanchu A. Adverse drug reactions to nonnucleoside reverse transcriptase inhibitor-based antiretroviral regimen: a 24-week prospective study. J Int Assoc Physicians AIDS Care. 2009;8(5):318-322.

72. Adkins JC, Noble S. Efavirenz. Drugs. 1998;56(6):1055-1064.

73. Bickel M, Stephan C, Rottmann C, et al. Severe CNS side-effect and persistent high efavirenz plasma levels in a patient with $\mathrm{HIV} / \mathrm{HCV}$ coinfection and liver cirrhosis. Scand J Infect Dis. 2005;37:520-522.

74. Gutiérrez F, Navarro A, Padilla S, et al. Prediction of neuropsychiatric adverse events associated with long-term efavirenz therapy, using plasma drug level monitoring. Clin Infect Dis. 2005;41(11):1648-1653.
75. Treisman GJ, Kaplin AI. Neurologic and psychiatric complications of antiretroviral agents. AIDS. 2002;16(9):1201-1215.

76. Blanch J, Corbella B, García F, Parellada E, Gatell JM. Manic syndrome associated with efavirenz overdose. Clin Infect Dis. 2001;33(2): 270-271.

77. Lochet P, Peyriere H, Lotthé A, Mauboussins JM, Delmas B, Reynes J. Long-term assessment of neuropsychiatric adverse reactions associated with efavirenz. HIV Med. 2003;4(1):62-66.

78. Marzolini C, Telenti A, Decosterd LA, Greub G, Biollaz J, Buclin T. Efavirenz plasma levels can predict treatment failure and central nervous system side effects in HIV-1-infected patients. AIDS. 2001;15(1): $71-75$.

79. Hasse B, Günthard F, Bleiber G, Krause M. Efavirenz intoxication due to slow hepatic metabolism. Clin Infect Dis. 2005;40(3):e22-e23.

80. Cohen CJ, Molina JM, Cahn P, et al. Efficacy and safety of rilpivirine (TMC278) versus efavirenz at 48 weeks in treatment-naïve HIV-1infected patients: pooled results from the phase 3 double-blind randomized ECHO and THRIVE trials. J Acquir Immune Defic Syndr. 2012; 60(1):33-42.

81. Anthonypillai C, Gibbs JE, Thomas SA. The distribution of the antiHIV drug, tenofovir (PMPA), into the brain, CSF and choroid plexuses. Cerebrospinal Fluid Res. 2006;3(1):1.

82. Best B, Letendre S, Koopmans P, et al. Low cerebrospinal fluid concentrations of the nucleotide HIV reverse transcriptase inhibitor, tenofovir. J Acquir Immune Defic Syndr. 2012;59(4):376-381.

83. Gallant JE, DeJesus E, Arribas J, et al. Tenofovir DF, emtricitabine, and efavirenz vs zidovudine, lamivudine, and efavirenz for HIV. $N$ Engl J Med. 2006;354(3):251-260.

84. Allavena C, Le Moal G, Michau C, Chiffoleau A, Raffi F. Neuropsychiatric adverse events after switching from an antiretroviral regimen containing efavirenz without tenofovir to an efavirenz regimen containing tenofovir: a report of nine cases. Antivir Ther. 2006;11(2): 263-265.

85. Rotger M, Colombo S, Furrer H, Décosterd L, Buclin T, Telenti A. Does tenofovir influence efavirenz pharmacokinetics? Antivir Ther. 2007;12(1):115-118.

86. HIV-druginteractions.org. Tenofovir efavirenz interaction report. Available from: http://www.hiv-druginteractions.org/interactions.aspx. Accessed September 1, 2012.
Neurobehavioral HIV Medicine

\section{Publish your work in this journal}

Neurobehavioral HIV Medicine is an international, peer-reviewed, open access journal focusing on advances in research in HIV/ AIDS, with specific reference to the neurological, psychiatric and behavioral consequences of the disease, concomitant infections and specific antiretroviral therapy. The manuscript

\section{Dovepress}

management system is completely online and includes a very quick and fair peer-review system, which is all easy to use. Visit http://www.dovepress.com/testimonials.php to read real quotes from published authors. 